\title{
DESCRIPTIVE CLASSES OF SETS IN NONSEPARABLE SPACES
}

\author{
PETR HOLICKÝ
}

\section{INTRODUCTION}

The classical descriptive set theory is devoted to the study of descriptive classes of sets in Polish spaces. There are many deep results which found applications, e.g., in real analysis or functional analysis. Several books and survey papers contain essential parts of this theory, see [49], [2], [48], [54], [63]. One of the key tools for the study of Borel sets in Polish spaces are analytic spaces.

There are several attempts to transfer parts of the theory to more general spaces. Among them two seem to form a satisfactorily rich theory $(K$-analytic spaces and absolute Souslin sets in metrizable spaces) and, in particular the first one, found interesting applications in the study of nonseparable Banach spaces and weak topologies on them. As representative surveys may serve [8] and [44]. Since these results enable to study just Lindelöf or metrizable topologies, some further extensions, inspired by the previous ones, were made by R.W. Hansell. There is a survey paper on generalized analytic spaces (on the "descriptive topology"), which points out some of key results, written by Hansell, see [22], cf. also [28], [32].

We start our survey by a choice of facts and problems concerning the descriptive classes of sets in metrizable spaces. We point out in particular some recent results concerning the mappings which preserve some descriptive properties and we list several still open problems which seem to be quite essential for the full understanding of the theory. Many of them are closely related and have a positive answer under an axiom of W.G.Fleissner (which is consistent with ZFC provided the existence of a supercompact cardinal is consistent).

Then we are going to discuss descriptive classes of sets in Tychonoff spaces. An important feature is that there are several natural notions of analyticity. They define classes of generalized analytic spaces which are ordered by the inclusion, but there is no unified attitude to all of them. The classes coincide within metrizable spaces and some results may be received repeating the methods used for metrizable spaces. However, many problems are specific. We recall some old and some more recent results, and we point out some open questions which seem to make the extension of the classical descriptive set theory and its applications to functional analysis not yet sufficiently complete.

We are aware that we cannot cover all related results and problems. For example, we mention only a few results concerning the Borel classes of sets and mappings, and we do so mainly when applying them to results on the $\sigma$-algebra of all Borel

\footnotetext{
2000 Mathematics Subject Classification. 54H05. sets.

Key words and phrases. absolute Souslin spaces, bimeasurable mappings, generalized Borel

The work is a part of the research project MSM 0021620839 financed by MSMT and partly supported by IAA100190901.
} 
sets. We shall also omit the part of the theory built by Z. Frolík and Holický [12], [14], [13], and the one built by Hansell, J.E. Jayne, and C.A. Rogers [24], [25], which covered the common parts of the theory of $K$-analytic spaces and Souslin subsets of complete metric spaces recalled separately below.

Our exposition does not follow neither the most natural order to prove the presented results nor the order of their historical appearance. We want to stress different aspects starting with the descriptions of absolute Souslin spaces as generalizations of corresponding complete spaces and adding gradually the other possible descriptions and several results and problems connected to them.

Let us recall first some notation which we shall often use. Throughout the paper, all topological spaces are assumed to be Tychonoff spaces. We use the notation $\mathcal{G}=\mathcal{G}(X), \mathcal{F}=\mathcal{F}(X), \mathcal{B}=\mathcal{B}(X), \mathcal{H}=\mathcal{H}(X)$ for the families of all open, closed, Borel, resolvable sets in a topological space $X$, respectively. Let us recall that $H \subset X$ is resolvable in $X$ if for every nonempty $F \subset X$ either $F \cap H$ or $F \backslash H$ has a nonempty relative interior in $F$. A set $S \subset X$ is the result of a Souslin operation applied to sets from a class of sets $\mathcal{C}$ if

$$
S=\bigcup_{\nu \in \mathbb{N}^{\mathbb{N}}} \bigcap_{n \in \mathbb{N}} C_{\nu \mid n}
$$

where $\nu \mid n$ denotes the restriction of $\nu$ to the first $n$ coordinates and $C_{\nu \mid n} \in \mathcal{C}(X)$ for every $\nu \mid n \in \mathbb{N}^{n}$. Let $\mathcal{S}(\mathcal{C}(X))$ stand for the sets which are results of the Souslin operation applied to sets from $\mathcal{C}(X)$ in a topological space $X$ (here $\mathcal{C}$ stands, e.g., for the class of closed, Borel, or resolvable sets in topological spaces). In any metrizable space $X, \mathcal{S}(\mathcal{F}(X))=\mathcal{S}(\mathcal{B}(X))=\mathcal{S}(\mathcal{H}(X))$ and we call the elements of $\mathcal{S}(\mathcal{F}(X))$ just Souslin sets in $X$.

For a mapping $f: X \rightarrow Y$ and a class of sets $\mathcal{C}$ as above we say here that $f$ is $\mathcal{C}$ measurable if $f^{-1}(\mathcal{C}(Y)):=\left\{f^{-1}(H): H \in \mathcal{C}(Y)\right\} \subset \mathcal{C}(X)$. We say that $f: X \rightarrow Y$ is $\mathcal{C}$ bimeasurable if $f$ is $\mathcal{C}$ measurable and $f$ takes sets of $\mathcal{C}(X)$ to sets of $\mathcal{C}(Y)$.

We say that $f: X \rightarrow Y$ preserves $\mathcal{P}$ (indexed) families, for some property $\mathcal{P}$ of (indexed) families of subsets of Tychonoff spaces, if $f(\mathcal{A}):=\{f(A): A \in \mathcal{A}\}$ (indexed by the sets $A$ ) has $\mathcal{P}$ in $Y$ if $\mathcal{A}$ has $\mathcal{P}$ in $X$.

\section{An EXTENSION OF CLASSICAL DESCRIPTIVE SET THEORY TO METRIZABLE TOPOLOGICAL SPACES}

We are going to study some "absolute descriptive classes within metrizable spaces" now. We say that a metrizable space $X$ is absolute $\mathcal{P}$ within metrizable spaces if it belongs to the class $\mathcal{P}$ in any metrizable space to which it is homeomorphically embedded. We shall omit the words "within metrizable spaces" sometimes in this section.

2.1. Completely metrizable spaces. Let us recall several characterizations of complete metrizability.

Theorem 2.1. The following statements on a topological space $X$ are equivalent:

(a) $X$ is completely metrizable.

(b) $X$ is metrizable and absolute $\mathcal{G}_{\delta}$ within metrizable spaces.

(c) $X$ is $G_{\delta}$ in a completely metrizable space.

If $X$ as above is moreover separable, it is Polish. 
These are well-known facts which can be found, e.g., in [4, Theorems 4.3 .23 and 4.3.24].

We also have a description of completely metrizable spaces in terms of a sequence of covers. We use the following notions.

A family $\mathcal{D}$ of sets in $X$ is discrete if $X$ can be covered by open sets such that each of them has nonempty intersection with at most one element of $\mathcal{D}$. It is $\sigma$-discrete if $\mathcal{D}=\bigcup_{n \in \mathbb{N}} \mathcal{D}_{n}$ and each $\mathcal{D}_{n}$ is discrete.

A family $\mathcal{R}$ of subsets of $X$ is a refinement of a family $\mathcal{E}$ if, for every $R \in \mathcal{R}$, there is $E \in \mathcal{E}$ such that $R \subset E$ and $\bigcup \mathcal{E}=\bigcup \mathcal{R}$. We say that $\mathcal{N}$ is a network of a family $\mathcal{E}$ of sets if $E=\bigcup\{N \in \mathcal{N}: N \subset E\}$ for every $E \in \mathcal{E}$.

We say that a sequence of covers $\mathcal{C}_{n}, n \in \mathbb{N}$, of a topological space $X$ is $a$ complete sequence of covers if $\bigcap\{\bar{F}: F \in \mathcal{F}\} \neq \emptyset$ for every filter $\mathcal{F}$ of subsets of $X$ with $\mathcal{F} \cap \mathcal{C}_{n} \neq \emptyset$ for every $n \in \mathbb{N}$ ("every $\left(\mathcal{C}_{n}\right)$-Cauchy filter has an accumulation point"). Note that replacing each cover $\mathcal{C}_{n}$ of a complete sequence of covers by any refinement $\mathcal{C}_{n}^{*}$, then the sequence of covers $\mathcal{C}_{n}^{*}$ is complete again. This is clear from the definition but often very useful when working with complete sequences of covers.

Theorem 2.2. A (separable) metrizable space $X$ is completely metrizable (Polish) if and only if there is a complete sequence of open covers of $X$, or equivalently, of $\sigma$-discrete open covers of $X$.

Let $\mathcal{C}_{n}$ be the cover of $X$ by open balls of diameter $\frac{1}{n}$ with respect to a complete metric on $X$. Then $\mathcal{C}_{n}$ 's obviously form a complete sequence of open covers. Due to paracompactness of $X$ each open cover $\mathcal{C}_{n}$ of the complete sequence in the theorem may be refined by a $\sigma$-discrete open refinement $\mathcal{C}_{n}^{*}$.

Having a complete sequence of open covers $\mathcal{C}_{n}$ of $X$ and $X$ embedded into some complete metric space $(\widehat{X}, \rho)$, we can refine each cover $\mathcal{C}_{n}$ of $X$ by a cover $\mathcal{C}_{n}^{*}$ consisting of open balls of $\rho$-diameter less than $\frac{1}{n}$ and notice that $X=\bigcap_{n \in \mathbb{N}} \bigcup\{\widehat{B}$ : $\left.B \in \mathcal{C}_{n}^{*}\right\}$, where $\widehat{B}=B_{\widehat{X}}(x, r)$ if $B=B_{X}(x, r)$. For more general statement and references see Theorem 3.1 in Section 3.1.

Every completely metrizable space can be parametrized in a particular way due to [17, Theorem 5.6].

Theorem 2.3. Every completely metrizable space $X$ is the image of a one-to-one continuous mapping $f: F \rightarrow X$, where $F$ is a closed subset of $D^{\mathbb{N}}, D$ is a discrete space, and $f$ preserves families with a $\sigma$-discrete network.

Since $f$ is one-to-one, $f(\mathcal{D})$ is disjoint for every discrete $\mathcal{D}$ and the existence of a $\sigma$-discrete network is equivalent to the existence of a $\sigma$-discrete refinement.

2.2. Souslin and discrete-Borel sets in completely metrizable spaces. A quite complete theory was based and essentially developed by Hansell (the fundamental work [15] was followed by a number of further papers by Hansell, e.g., [17], [16], [18]) who continued in the work of A.H. Stone. We recall the basic notions and statements which coincide with the classical ones in separable metrizable spaces and which enable to get many results similar to those of the classical descriptive set theory. We are going to point out some of them together with related open problems. Let us recall several characterizations of absolute Souslin and absolute bi-Souslin spaces, i.e., spaces which are Souslin together with their complement in any metrizable embedding. 
Theorem 2.4. The following statements are equivalent for a metrizable space A:

(a) A is absolute Souslin within metrizable spaces.

(b) $A$ is a Souslin subset of a completely metrizable space.

(c) $A$ is the projection of a completely metrizable subspace $C$ of $A \times \mathbb{N}^{\mathbb{N}}$.

(d) A admits a complete sequence of $\sigma$-discrete covers.

The equivalence of (a) and (b) is an easy consequence of Lavrentieff's theorem (see [4, Theorem 4.3.21]). The equivalence of (b) and (c) follows from the wellknown characterization of Souslin sets. The equivalence of $(\mathrm{d})$ and $(\mathrm{b})$ is a particular case of [14, Theorem 4.1], since topologically discrete families have metrically $\sigma$ discrete refinements in $A$ with respect to an arbitrarily chosen metric (cf. [15, Lemma 2]).

The spaces $A$ with the properties of the previous theorem are called analytic if they are separable. They are most often defined as continuous images of Polish spaces. We shall discuss continuous parametrizations of absolute Souslin spaces later. Also the nonseparable spaces of the above theorem are often called analytic. To avoid ambiguity, we abbreviate absolute Souslin within metrizable spaces by metric-analytic here. Every completely metrizable space is clearly metric-analytic. The property (c) above gives some kind of parametrization which however does not make clear how to deduce any result on the preservation of metric-analytic spaces by particular continuous mappings.

The first Luzin separation principle and many of its corollaries found its counterpart in the realm of nonseparable metrizable spaces. It explains why Souslin sets can be viewed as a tool to study Borel sets in Polish spaces. However, in nonseparable spaces we get an extension of the classical theory for a larger $\sigma$-algebra of sets called, e.g., "extended Borel sets" by Hansell. We use another name to distinguish these sets from the elements of $\sigma$-algebras arising similarly when studying the topological variants of absolute Souslin spaces. By discrete-Borel sets in a metrizable space $X$ we understand the elements of the smallest $\sigma$-algebra which contains Borel subsets of $X$ and which is closed with respect to the unions of its discrete subfamilies. Since discrete families of sets in separable metrizable spaces are countable, the $\sigma$-algebras of Borel and discrete-Borel sets coincide in separable metrizable spaces. We get the following relation between absolute bi-Souslin and absolute discrete-Borel sets. It is a particular case of a separation principle and the subsequent theorem is one of many results relying on it.

Theorem 2.5. A subset $B$ of a metric-analytic space $A$ is discrete-Borel in $A$ if and only if both $B$ and $A \backslash B$ are metric-analytic (or, equivalently, Souslin in $A$ ).

This result can be deduced from some more general once, e.g., [13, Theorem 4.4(a)], or it follows from two results of Hansell as explained in [22, Theorem 4.5]. The proofs use the parametrizations which we shall discuss later. However, they could be easily rewritten with a use of the complete sequences of $\sigma$-discrete covers. We shall see why this observation may be of some interest for the study of a class of generalized analytic spaces ("( $\sigma$-isolated)-K-complete spaces") below.

The images of Polish spaces by one-to-one continuous mappings are sometimes called Luzin spaces. In this particular case, they coincide with separable absolute Borel sets (within metrizable spaces). We shall see that absolute discrete-Borel spaces can be characterized by some continuous one-to-one parametrizations. The 
condition (c) of the following theorem offers one such parametrization. Let us therefore use the abbreviation metric-Luzin for absolute discrete-Borel spaces within metrizable spaces.

Theorem 2.6. The following statements are equivalent for a metrizable space $L$ :

(a) $L$ is absolute discrete-Borel within metrizable spaces.

(b) $L$ is discrete-Borel in some completely metrizable space.

(c) $L$ is the one-to-one projection of a completely metrizable subspace of $L \times \mathbb{N}^{\mathbb{N}}$.

While the equivalence of (a) and (b) follows naturally by the Lavrentieff theorem, an explanation of the equivalence of (c) and (a) is in [34, Theorem 2.2] and uses some more results of the theory.

We get a number of results concerning discrete-Borel sets (and discrete-Borel measurable and bimeasurable mappings), while the analogical study of Borel sets (and mappings) in nonseparable metrizable spaces is often an open problem. We shall mention several such problems and positive results.

2.3. Measurable mappings. Let $f: X \rightarrow Y$ be a Souslin measurable mapping between metrizable spaces $X$ and $Y$. Then $Y$ has a basis $\mathcal{V}=\bigcup_{n \in \mathbb{N}} \mathcal{V}_{n}$, where $\mathcal{V}_{n}$ are discrete families of open sets in $Y$. So each $\mathcal{U}_{n}=\left\{f^{-1}(V): V \in \mathcal{V}_{n}\right\}$ is a disjoint family with the property that $\bigcup \mathcal{W}$ is Souslin for every $\mathcal{W} \subset \mathcal{U}$. The families with this property are called Souslin additive. (If Souslin is replaced by any other class $\mathcal{C}$ of sets, we speak about $\mathcal{C}$ additive families.)

Let us recall a deep fundamental result of Hansell [15, Theorem 2], and its improvement of J. Kaniewski and R. Pol [47, Theorem 1].

Lemma 2.7. A disjoint (point-finite) Souslin additive family in a completely metrizable space has a $\sigma$-discrete network.

As a corollary we observe

Proposition 2.8. A mapping $f: X \rightarrow Y$ of a metric-analytic space $X$ onto a metrizable space $Y$ is Souslin (discrete-Borel, Borel) measurable if and only if $f^{-1}(G)$ is Souslin (discrete-Borel, Borel) for every open set $G$ in $Y$.

Let the obviously necessary condition be satisfied. Since all Borel sets and Souslin sets in metrizable spaces are generated from closed sets by some of the operations of taking complements, unions, and intersections, which commute with the operation of preimages, the results for Borel and Souslin are obvious. The only nontrivial fact is that preimages of unions of discrete families $\mathcal{E}$ of sets with discrete-Borel preimages are discrete-Borel. Indeed, $\mathcal{D}=f^{-1}(\mathcal{E})$ is Souslin additive and disjoint. Therefore there is a $\sigma$-discrete network $\mathcal{N}$ of $\mathcal{D}$ by Lemma 2.7. For every $N \in \mathcal{N}$ consider the only $D \in \mathcal{D}$ with $N \subset D$. Replacing $N$ by $N^{*}=\bar{N} \cap D$ we get a $\sigma$-discrete network (here refinement is equivalent) $\mathcal{N}^{*}=\left\{N^{*}: N \in \mathcal{N}\right\}$ which consists of discrete-Borel sets. Thus $\bigcup \mathcal{D}=\bigcup \mathcal{N}^{*}$ is discrete-Borel.

This proposition is just a sample of many applications of Lemma 2.7 to measurable mappings. A lot of classical results may be extended due to it (see [15] and the other forthcoming papers of Hansell quoted above).

Let us point out another important result on disjoint Borel additive families [57, Theorem] (see also [5, Theorem 2.6], or [19, Theorem 1], for the case of point-finite Borel additive families). 
Lemma 2.9. Let $\mathcal{A}$ be a point-finite Borel additive family of subsets of a metrizable space. Then there is an additive Borel class $\mathcal{C}$ such that $\mathcal{A} \subset \mathcal{C}$.

This together with Hansell's Lemma 2.7 and [17, Lemma 7] gives (see, e.g., [19, Corollary 1])

Theorem 2.10. Every Borel measurable mapping $f: X \rightarrow Y$ of a metric-analytic space $X$ to a metrizable space $Y$ has a Borel class, i.e., there is and additive Borel class $\mathcal{A}$ such that $f^{-1}(\mathcal{G}(Y)) \subset \mathcal{A}$.

2.4. Parametrizations of absolute Souslin sets and Souslin bimeasurable mappings. While classical analytic spaces are characterized as continuous images of Polish spaces, and so they are preserved by continuous mappings, any topological space $X$ is a continuous one-to-one image of a completely metrizable space, namely the identity on $X$ considered as a mapping of $X$ endowed with the discrete topology. Thus it is not evident which mappings preserve metric-analytic spaces. This should be however one of the most important conclusions of any extension of the notion of analytic spaces.

Analyzing condition (d) of Theorem 2.4, we may come to the following characterizations of metric-analyticity in terms of continuous parametrizations.

Theorem 2.11. The following statements about a metrizable space $A$ are equivalent:

(a) $A$ is metric-analytic.

(b) There is a discrete space $D$, a closed $F \subset D^{\mathbb{N}}$, and a continuous mapping $f: F \rightarrow A$ such that $f(F)=A$ and $f$ preserves families with a $\sigma$-discrete refinement.

(c) There is a discrete space $D$, a closed $F \subset D^{\mathbb{N}}$, and a continuous mapping $f: F \rightarrow A$ such that $f(F)=A$ and $f$ preserves point-countable indexed families with a $\sigma$-discrete network.

This can be proved following the proof of the corresponding implication of [14, Theorem 4.1] just for the metrizable spaces. The existence of the stronger parametrizations of metric-analytic spaces from (c) was proved already in [17, Theorem 4.1]. Notice that (c) of Theorem 2.4 gives us a parametrization which is continuous and preserves point-countable indexed families with a $\sigma$-discrete network defined on the completely metrizable $C$. So it is sufficient to use Theorem 2.3.

As an immediate corollary we get the following result on preservation of metricanalyticity in case of continuous mappings $f$ which preserve families with a $\sigma$ discrete refinement.

Theorem 2.12. Let $f: X \rightarrow Y$ be a Souslin measurable mapping preserving families with a $\sigma$-discrete refinement of a metric-analytic space $X$ onto a metrizable space $Y$. Then $Y$ is metric-analytic and $f: X \rightarrow Y$ is Souslin bimeasurable.

If $f$ is continuous, we may parametrize $X$ (or $S \in \mathcal{S}(\mathcal{F}(X))$ ) by a $\varphi: F \subset D^{\mathbb{N}} \rightarrow$ $S$ as in Theorem 2.11(b), compose it with $f$, and use Theorem 2.11 again.

The assumption of continuity could be relaxed to the weaker assumption of Souslin measurability by standard methods. In a more general setting it can be found in [13, Theorem 4.4(a)]. It can be also proved following [17, Theorem 7.3]. However, we have to replace the application of [17, Corollary 4.2] by Theorem 2.12 and the application of $[17$, Lemma 6.1$]$ by $[14$, Lemma $2.5(\mathrm{~b})]$. We want to formulate 
explicitly some still open problems concerning the question whether the condition of preserving the existence of $\sigma$-discrete refinements can be relaxed. On the other hand, we want to know whether some formally stronger conditions on the mappings are also necessary.

Let us consider a Souslin bimeasurable mapping $f: X \rightarrow Y$ of a metric-analytic space $X$ onto a metric-analytic space $Y$. If $\mathcal{D}$ is a discrete family of Souslin subsets of $X$, then $\mathcal{D}$ is Souslin additive. So $\mathcal{E}=f(\mathcal{D})$ is also Souslin additive. In fact, we can deduce more. Consider any Souslin $F_{D} \subset f(D)$ for $D \in \mathcal{D}$. Then $F_{D}=$ $f\left(f^{-1}\left(F_{D}\right) \cap D\right)$. The sets $f^{-1}\left(F_{D}\right) \cap D$ form a discrete family of Souslin sets and so their union is again Souslin. Hence $\bigcup\left\{F_{D}: D \in \mathcal{D}\right\}$ is Souslin in $Y$.

Problem 2.13. Let $\mathcal{E}$ be a family of Souslin sets in a completely metrizable space $Y$ such that for every choice of Souslin $S_{E} \subset E, E \in \mathcal{E}$, the union $\bigcup\left\{S_{E}: E \in \mathcal{E}\right\}$ is Souslin.

(a) Does $\mathcal{E}$ have a $\sigma$-discrete refinement?

(b) Does $\mathcal{E}$ have a $\sigma$-discrete network?

(c) Does there exist a $\sigma$-discrete set $D$ such that $\{E \backslash D: E \in \mathcal{E}\}$ is pointcountable (in other words, is $\mathcal{E}$ point-countable outside of $D$ )?

The two requirements (b) and (c) on a family $\mathcal{E}$ are abbreviated by $\mathcal{E}$ is almost discretely $\sigma$-decomposable in [26]. This property is very useful because taking any subset of each element of such a family, we get a family with the same property. It follows immediately from the fact that every point-countable family which has a $\sigma$-discrete network is " $\sigma$-discretely decomposable" (see [15] for the definition of $\sigma$-discretely decomposable families and [53, Lemma 3.2]). This explains also the name chosen for almost discretely $\sigma$-decomposable families. Such a heredity does not hold for families with a $\sigma$-discrete network or $\sigma$-discrete refinement in general.

The positive solution for some of the properties (a) - (c) of $\mathcal{E}$ would give us that the Souslin bimeasurable mapping $f$ above takes necessarily discrete families of Souslin sets to families $\mathcal{E}$ with the corresponding property from (a), (b), or (c). Therefore we ask the following questions.

Problem 2.14. Let $f: X \rightarrow Y$ be a Souslin measurable (or just discrete-Borel measurable) mapping of a completely metrizable space $X$ onto a metrizable space $Y$. Let $f(\mathcal{D})$ have a $\sigma$-discrete refinement whenever $\mathcal{D}$ is a discrete family of Souslin sets in $X$.

(a) Is then $Y$ metric-analytic?

(b) Does $f$ preserve families with a $\sigma$-discrete refinement?

(c) Does $f$ preserve almost discretely $\sigma$-decomposable families?

The positive answer to (b) would give us a positive answer to (a). Let us remark that (c) is equivalent with the property that $f$ takes discrete families to almost discretely $\sigma$-decomposable families, similarly as for families with a $\sigma$-discrete network or refinement. Souslin measurability implies that preimages of discrete-Borel, so bi-Souslin, sets in $Y$ are bi-Souslin in $X$, and thus discrete-Borel by Theorem 2.5.

Lemma 2.7 gives as a consequence the answer for particular classes of mappings:

Theorem 2.15. Let $f: X \rightarrow Y$ be a Souslin measurable mapping with relatively compact fibers between metric-analytic spaces $X$ and $Y$ such that $f(\mathcal{F}(X)) \subset$ $\mathcal{S}(\mathcal{F}(Y))$. Then $f$ preserves families with a $\sigma$-discrete network. In particular, $f: X \rightarrow Y$ is Souslin bimeasurable. 
Indeed, if $\mathcal{D}$ is a discrete family of subsets of $X$, then $\{\bar{D}: D \in \mathcal{D}\}$ is discrete, so "closed additive". Then $\{f(\bar{D}): D \in \mathcal{D}\}$ is Souslin additive and point-finite by our assumptions. Now we may apply Lemma 2.7. If $A$ is Souslin in $X$, then it is metric-analytic, the restriction of $f$ to $A$ preserves families with a $\sigma$-discrete network, and we may apply Theorem 2.12 .

Let us remark that the previous theorem applies in particular if $f$ is a perfect mapping. We shall see that for some classes of generalized analytic topological spaces we are able to prove the preservation by perfect mappings also in cases were we have no analogy to Theorem 2.15 and to Lemma 2.7.

Fleissner [5, Theorem 5.3] proved that if the existence of a supercompact cardinal is consistent with $\mathrm{ZFC}$, then an axiom $\left(\mathrm{SC} \omega_{2}\right)$ is consistent with $\mathrm{ZFC}$, which gives a positive answer to our above problems. It follows from the results of Fleissner that, under $\left(\mathrm{SC} \omega_{2}\right)$, a mapping $f$ of a metric space $X$ onto a metric space $Y$, which takes discrete sets to $\sigma$-discrete sets, takes discrete families of sets to almost discretely $\sigma$-decomposable families (cf. [26, Theorem 2(a)]), equivalently, it preserves almost discretely $\sigma$-decomposable families.

Let us consider $f: X \rightarrow Y$ from Problem 2.14 (except for the completeness of $X)$. Of course, any discrete set $D$ can be viewed as a discrete family of singletons, which are Souslin sets, and so the singletons which are their images form a family which has a $\sigma$-discrete refinement. This means just the $\sigma$-discreteness of $f(D)$. Thus under $\left(\mathrm{SC} \omega_{2}\right)$ the answers to the above Problem 2.14 are affirmative.

The solutions of Problem 2.13 can be also deduced under $\left(\mathrm{SC} \omega_{2}\right)$ from Fleissner's results. Consider, e.g., the discrete sum $X$ of the elements of $\mathcal{E}$. Define $f: X \rightarrow Y$ as the identity on each $E \in \mathcal{E}$. Now $f$ takes discrete sets $D$ to sets with all subsets Souslin in $Y$ by the assumption. Using Lemma 2.7, the sets $f(D)$ are $\sigma$ discrete. Now the positive solution of Problem 2.14(c) gives the positive solution of Problem 2.13. The family $\mathcal{E}$ is almost discretely $\sigma$-decomposable.

Theorem 2.16 (under $\left.\left(\mathrm{SC} \omega_{2}\right)\right)$. Let $f: X \rightarrow Y$ be a Souslin measurable mapping of a metrizable space $X$ to a metrizable space $Y$ which takes closed sets in $X$ to metric-analytic sets. Then $f$ preserves almost discretely $\sigma$-decomposable families, and so it preserves $\sigma$-discretely refinable families. Thus the sufficient condition of Theorem 2.12 is also necessary.

We shall discuss below the possibility to show that every discrete-Borel bimeasurable mapping between complete metric spaces can be decomposed to a countable $(\sigma$-discrete) family of Borel isomorphisms and a mapping with a $\sigma$-discrete range. Since every projection along an analytic space is Souslin bimeasurable, we cannot await much more than showing that every Souslin bimeasurable mapping is related to such a projection up to a mapping with a $\sigma$-discrete range. We can view the problem of preservation of almost discretely $\sigma$-decomposable families by metric-analytic bimeasurable mappings as an attempt to relate all metric-analytic bimeasurable mappings to projections of metric-analytic spaces along $\mathbb{N}^{\mathbb{N}}$ due to the following consequence of [37, Theorem 3.2].

Proposition 2.17. Let $f: X \rightarrow Y$ be a mapping of a metric-analytic space $X$ to a completely metrizable space $Y$. Then $f$ is Souslin measurable and preserves point-countable indexed families with a $\sigma$-discrete network if and only if there is a discrete-Borel isomorphism $h$ of $X$ onto a metric-analytic set $H \subset \mathbb{N}^{\mathbb{N}} \times Y$ such that $f=p \circ h$, where $p$ is the projection of $H$ to $Y$ restricted to $H$. 
We prove first the necessity of the existence of $h$. The space $X$ is discreteBorel isomorphic to a metric-analytic subset $A$ of $D^{\mathbb{N}}$ for some discrete $D$ by $[17$, Theorems 5.6 and 6.7] applied to the completion of $X$. Thus we may assume that $X=A \subset D^{\mathbb{N}}$. Let $\pi_{X}$ be the projection of $X \times Y$ to $X$ and $\psi$ be the inverse mapping to the restriction of $\pi_{X}$ to the metric-analytic graph $G \subset X \times Y \subset D^{\mathbb{N}} \times Y$ of $f$. Then $\psi$ is a discrete-Borel isomorphism ( $f$ is metric-analytic measurable, so $\psi$ is metric-analytic measurable and it obviously takes discrete families to discrete families in $G$; since it is one-to-one, it is a discrete-Borel isomorphism by [17, Theorem 7.4]). Applying the discrete-Borel isomorphism $\Psi$ of [37, Theorem 3.2] to the graph $G$ of $f$, we may put $h=\Psi \circ \psi$. Finally, we have that $f=p \circ h$.

Conversely, $p$ is continuous and takes metric-analytic sets in $\mathbb{N}^{\mathbb{N}} \times Y$ to metricanalytic sets in $Y$ (the projections of Souslin sets along $\mathbb{N}^{\mathbb{N}}$ are Souslin in $Y$ ). The mapping $h$ is a discrete-Borel isomorphism, and so also a metric-analytic measurable mapping (we may use [17, Theorem 6.7]) which preserves point-countable families with $\sigma$-discrete network, of the metric-analytic $G$ onto $H$. This concludes the proof.

Continuous mappings $f$ with the factorization of the same form and with $h$ a topological embedding were studied by Frolík in [11] under the name $S$-maps.

If the Souslin measurable mapping $f$ preserves almost discretely $\sigma$-decomposable families as in Theorem 2.15, we can find a $\sigma$-discrete set $D \subset Y$ such that $f \uparrow$ $\left(X \backslash f^{-1}(D)\right)$ preserves point-countable indexed families with a $\sigma$-discrete network. It is not difficult to check that a $\sigma$-discrete set $D$ from the definition of almost discrete $\sigma$-decomposability of $f(\mathcal{D})$, where $\mathcal{D}$ is an arbitrary $\sigma$-discrete base of the topology of $X$, works. So $f \uparrow\left(X \backslash f^{-1}(D)\right)$ can be viewed as a projection along $\mathbb{N}^{\mathbb{N}}$ composed with a discrete-Borel isomorphism as in Theorem 2.17 for a fixed $\sigma$-discrete $D \subset Y$ if the conclusion of Theorem 2.16 holds.

2.5. Preservation of discrete-Borel sets. The characterization of discrete-Borel sets as bi-Souslin in metric-analytic spaces (Theorem 2.5) and Theorem 2.12 give the following sufficient condition for a mapping to be relatively discrete-Borel bimeasurable.

Theorem 2.18. Let $X$ be metric-analytic, $Y$ a metrizable space, and $f: X \rightarrow Y a$ discrete-Borel measurable one-to-one mapping preserving families with a $\sigma$-discrete network. Then $f$ is discrete-Borel bimeasurable.

Let us recall that absolute discrete-Borel spaces within metrizable spaces are called metric-Luzin.

Theorem 2.19. A metrizable space $L$ is metric-Luzin if and only if $L$ is the image of a one-to-one continuous mapping $f: F \rightarrow L$, where $F$ is a closed subset of $D^{\mathbb{N}}$ for some discrete space $D$, and $f$ preserves the property of families of sets to have a $\sigma$-discrete refinement.

This result is in [17, Theorem 5.6] and it can be deduced from Theorems 2.3 and $2.6(\mathrm{c})$.

As a corollary we have

Theorem 2.20. The class of metric-Luzin spaces is preserved by one-to-one discrete-Borel measurable mappings which preserve the families with a $\sigma$-discrete refinement.

However, the requirements on the mappings to be one-to-one (and to preserve $\sigma$-discrete refinements) might seem to be too restrictive. 
Let us consider a continuous mapping $f: X \rightarrow Y$ between completely metrizable spaces and assume that $f$ takes discrete-Borel sets to discrete-Borel sets. Let $\mathcal{D}$ be a discrete family of discrete-Borel subsets of $X$ and $\prec$ be a strict well-ordering of $\mathcal{D}$. Consider $F_{D}=f(D) \backslash\left\{f\left(D^{\prime}\right): D^{\prime} \prec D\right\}$. Now, similarly as before Problem 2.13 above, the disjoint family $\left\{F_{D}: D \in \mathcal{D}\right\}$ is Souslin (even discrete-Borel) additive in $Y$. Thus it has a $\sigma$-discrete refinement, which is also a $\sigma$-discrete refinement of $f(\mathcal{D})$. This might inspire a question related to the above Problem 2.14(b). Again, under $\left(\mathrm{SC} \omega_{2}\right)$, Fleissner's results give the positive answer as above.

Problem 2.21. Let $\mathcal{E}$ be a family of discrete-Borel subsets of a completely metrizable space $Y$ such that for any choice of discrete-Borel sets $B_{E} \subset E, E \in \mathcal{E}$, the union $\bigcup\left\{B_{E}: E \in \mathcal{E}\right\}$ is discrete-Borel. Does $\mathcal{E}$ have a $\sigma$-discrete network? Is it point-countable outside of a $\sigma$-discrete set? (In other words, is it almost discretely $\sigma$-decomposable?)

The positive answer to this question would allow us to get strong results on the structure of discrete-Borel bimeasurable mappings.

Theorem 2.22 ([36, Theorem 3.1]). Let $X$ and $Y$ be complete metric spaces and $f: E \subset X \rightarrow Y$ be a discrete-Borel measurable mapping of a discrete-Borel set $E$ in $X$ to $Y$. Then the following are equivalent:

(a) $f$ is discrete-Borel bimeasurable and $f$ preserves almost discretely $\sigma$-decomposable families.

(b) $f(B)$ is discrete-Borel for every $G_{\delta}$ subset $B$ of $E$ and $f$ preserves almost discretely $\sigma$-decomposable families.

(c) $\left\{y \in Y: f^{-1}(y)\right.$ is not countable $\}$ is $\sigma$-discrete and $f \uparrow\left(X \backslash f^{-1}(S)\right)$ preserves families with a $\sigma$-discrete network.

(d) There are pairwise disjoint discrete-Borel subsets $E_{0}, E_{1}, \ldots$ of $X$ such that $E=\bigcup_{n=0}^{\infty} E_{n}, f\left(E_{0}\right)$ is $\sigma$-discrete, and $\left.f\right|_{E_{n}}$ is a discrete-Borel isomorphism for every $n \in \mathbb{N}$.

Note that the condition $f$ preserves almost discretely $\sigma$-decomposable families is fulfilled if $X$ is separable, and the theorem reduces to results of Luzin, Novikov, and of Purves in this case (cf. [41, Theorem 8]).

Under the Fleissner's axiom we have a positive solution of Problem 2.21 and it gives the following stronger formulation of the theorem.

Theorem 2.23 (under $\left(\mathrm{SC}_{2}\right)$, [36, Theorem 3.2]). Let $X$ and $Y$ be complete metric spaces and $f: E \subset X \rightarrow Y$ be a discrete-Borel measurable mapping of a discrete-Borel set $E$ in $X$ to $Y$. Then the following are equivalent:

(a) $f$ is discrete-Borel bimeasurable.

(b) $f(B)$ is discrete-Borel for every $G_{\delta}$ subset $B$ of $E$.

(c) $S=\left\{y \in Y: f^{-1}(y)\right.$ is not countable $\}$ is $\sigma$-discrete and $f \uparrow\left(X \backslash f^{-1}(S)\right)$ preserves families with a $\sigma$-discrete network.

(d) There are pairwise disjoint discrete-Borel subsets $E_{0}, E_{1}, \ldots$ of $X$ such that $E=\bigcup_{n=0}^{\infty} E_{n}, f\left(E_{0}\right)$ is $\sigma$-discrete, and $\left.f\right|_{E_{n}}$ is a discrete-Borel isomorphism for every $n \in \mathbb{N}$.

So another variant of Problem 2.21 can be formulated briefly.

Problem 2.24. Does Theorem 2.23 hold in ZFC? 
A positive answer in ZFC to the following question could be understood as a partial answer to the previous ones.

Problem 2.25. Let $f$ be a discrete-Borel bimeasurable mapping between completely metrizable spaces. Is $f$ Souslin bimeasurable?

Without any additional assumptions on the discrete-Borel bimeasurable mappings, we have only the following theorem on a $\sigma$-discrete decomposition of the mapping from [36, Corollary 2.2] instead of the countable decomposition from parts (d) of Theorems 2.22 and 2.23 .

Theorem 2.26. Let $X$ and $Y$ be complete metric spaces, $E$ be a discrete-Borel subset of $X$, and $f: E \rightarrow Y$ be a discrete-Borel measurable mapping. Then $f$ is discrete-Borel bimeasurable if and only if

(a) there is a $\sigma$-discrete subset $S$ of $Y$ and there is a $\sigma$-discrete partition $\mathcal{D}$ of $E \backslash$ $f^{-1}(S)$ to discrete-Borel sets such that $\left.f\right|_{D}$ is a discrete-Borel isomorphism for every $D \in \mathcal{D}$, and

(b) $\left\{B_{D}\right\}_{D \in \mathcal{D}}$ is discrete-Borel additive whenever $B_{D} \subset f(D)$ are discreteBorel.

The implication (c) implies (d) in Theorem 2.22 uses a decomposition from [34, Theorem 3.1] of Souslin subsets of $X \times \mathbb{N}^{\mathbb{N}}$ with countable sections.

We have a similar result for sets with $\sigma$-compact sections ([36, Theorem 3.3]), which is a generalization of theorems of V. Arsenin, K. Kunugui (see [48, Theorem 18.18]), J. Saint Raymond (see [59]), and Holický and M. Zelený (see [41, Main Theorem]).

Theorem 2.27. Let $X$ and $Y$ be complete metric spaces and $f: E \subset X \rightarrow Y$ be a discrete-Borel measurable mapping of a discrete-Borel set $E$ in $X$ to $Y$. Then the following are equivalent:

(a) $f(B)$ is discrete-Borel for every closed subset $B$ of $E$ and $f$ preserves almost discretely $\sigma$-decomposable families.

(b) $\left\{y \in Y: f^{-1}(y)\right.$ is not $\sigma$-compact $\}$ is $\sigma$-discrete and $f$ preserves almost discretely $\sigma$-decomposable families.

(c) There are discrete-Borel subsets $E_{0}, E_{1}, \ldots$ of $X$ such that $E=\bigcup_{n=0}^{\infty} E_{n}$, $f\left(E_{0}\right)$ is $\sigma$-discrete, and $\left.f\right|_{E_{n}}$ maps closed sets to discrete-Borel sets, and $\left.f\right|_{E_{n}}$ has compact fibers $\left(\left.f\right|_{E_{n}}\right)^{-1}(y), y \in Y$, for every $n \in \mathbb{N}$.

The variant of Theorem 2.26 with the axiom $\left(\mathrm{SC}_{2}\right)$ can be formulated similarly as Theorem 2.23 above ([36, Theorem 3.4]).

Inspired by a theorem of J.P. Burgess and G. Hillard (for the theorem and also for the definition of a hereditary co-analytic family of closed sets see [48, Theorem 35.43]), we may ask the following question.

Problem 2.28. Let $\mathcal{I}^{*}$ be a hereditary co-analytic family of closed subsets of $\mathbb{N}^{\mathbb{N}}$, $X$ be a completely metrizable space, and $B \subset X \times \mathbb{N}^{\mathbb{N}}$ be a (discrete-)Borel set with section $A_{x}, x \in X$, in $\mathcal{I}_{\sigma}^{*}$. Are there discrete-Borel sets $B_{n} \subset X \times \mathbb{N}^{\mathbb{N}}$ such that $B=\bigcup_{n \in \mathbb{N}} B_{n}$ and all sections $\left(B_{n}\right)_{x}, x \in X$, are in $\mathcal{I}^{*}$ ?

So for the particular cases of families of finite sets and of compact sets we have a positive answer (Theorems 2.22 and 2.27 above, or [34, Theorem 3.1] and [35, Theorem 3.1], to which their proofs are reduced in [36]). It seems that a crucial 
problem is the lack of a method which would replace the boundedness principle for co-analytic ranks (see [48, Theorem 35.22]).

There are also no results analogous to those of A. Louveau in [50] and [51] for Borel sets with sections of additive Borel classes in $X \times \mathbb{N}^{\mathbb{N}}$ with $X$ completely metrizable which would be much stronger than the result on the decomposition of sets with $\sigma$-compact sections.

There is still another result on preservation of metric-Luzin spaces under discreteBorel bimeasurable mappings $f: X \rightarrow Y$ which satisfy some condition in terms of "discrete-Borel classes" of subsets of $X$ and $Y$, see [33, Theorem 4.4]. Since we do not define the discrete-Borel classes here, we formulate only a particular case in Theorem 2.35 below.

2.6. Preservation of Borel sets. Following the decompositions of the previous section, we pose a problem.

Problem 2.29. Is a Borel measurable mapping $f: X \rightarrow Y$ between completely metrizable spaces $X$ and $Y$ Borel bimeasurable if and only if there are pairwise disjoint Borel sets $E_{0}, E_{1}, \ldots$ of $X$ such that $E=\bigcup_{n=0}^{\infty} E_{n}, f\left(E_{0}\right)$ is $\sigma$-discrete, and $\left.f\right|_{E_{n}}$ is a Borel isomorphism for every $n \in \mathbb{N}$ ? Do they map open sets to sets of a bounded Borel class?

Most answers are positive under the axiom $\left(\mathrm{SC} \omega_{2}\right)$ by Theorem 2.23. It is not clear that $E_{n}$ 's can be chosen Borel even for mappings which preserve almost discretely $\sigma$-decomposable families. The inverses to the Borel isomorphisms $\left.f\right|_{E_{n}}$ are of bounded class by Theorem 2.10. (We might also apply a deep theorem of D. Fremlin, Hansell, and H.J.K. Junnila [7] saying that a Borel isomorphism between metric spaces, not necessarily completely metrizable, has a bounded Borel class.)

Let us make some more elementary observations concerning the boundedness of the Borel class. We use the simple fact that a discrete family of Borel sets has a Borel union if and only if its elements are of bounded Borel class.

Lemma 2.30. Let $\mathcal{E}$ be an almost discretely $\sigma$-decomposable family in a completely metrizable space $X$ such that $\bigcup\left\{E^{*}: E \in \mathcal{E}\right\}$ is Borel for every choice of relatively closed subsets $E^{*}$ of $E \in \mathcal{E}$. Then $\mathcal{E}$ is $\mathcal{A}$-additive, where $\mathcal{A}$ is some additive Borel class.

Let $S$ be a $\sigma$-discrete set in $X$ such that the family $\mathcal{D}=\{E \backslash S: E \in \mathcal{E}\}$ is pointcountable with a $\sigma$-discrete network (in other words, discretely $\sigma$-decomposable). For any choice of relatively closed sets $(E \backslash S)^{*}$ in $E \backslash S$, we have $\bigcup\left\{(E \backslash S)^{*}: E \in\right.$ $\mathcal{E}\}=\bigcup\left\{\overline{(E \backslash S)^{*}} \cap(E \backslash S): E \in \mathcal{E}\right\}=\bigcup\left\{E \cap \overline{(E \backslash S)^{*}}: E \in \mathcal{E}\right\} \backslash S$. Since each $E \cap \overline{(E \backslash S)^{*}}$ is closed in $E$, the union $\bigcup\left\{E \cap \overline{(E \backslash S)^{*}}: E \in \mathcal{E}\right\}$ is Borel. The space $X \backslash S$ is completely metrizable. If the $\mathcal{D}$ belongs to some bounded additive Borel class then the same is true for $\mathcal{E}$, and so we may assume without loss of generality that $S$ is empty.

By [15, Lemma 7] it is enough to show that all elements of $\mathcal{E}$ are of a bounded additive Borel class. Let $\mathcal{N}=\bigcup_{n \in \mathbb{N}} \mathcal{N}_{n}$ be a network for $\mathcal{E}$ and all $\mathcal{N}_{n}$ be discrete. Assume that all elements of $\mathcal{N}$ are nonempty. Let us order the at most countable set of elements of $\mathcal{E}$ which contain an $N \in \mathcal{N}$ to a sequence and denote by $\mathcal{N}_{n}(E, k)$, $E \in \mathcal{E}, k \in \mathcal{N}$, the family of all elements $N$ of $\mathcal{N}_{n}$ such that $E$ is the $k$-th set containing $N$. Put $E_{n}^{*}(k)=E \cap \overline{\mathcal{U N}_{n}(E, k)}$ for $E \in \mathcal{E}$. Consider an arbitrary 
family $\mathcal{E}_{0} \subset \mathcal{E}$. By our assumption, the union $\bigcup\left\{E_{n}^{*}(k): E \in \mathcal{E}_{0}\right\}$ is Borel (empty set is closed in elements of $\left.\mathcal{E} \backslash \mathcal{E}_{0}\right)$. It follows that the discrete family $\left\{E_{n}^{*}(k)\right.$ : $E \in \mathcal{E}\}$ is Borel additive and so of a bounded additive Borel class $\alpha(n, k)$ for every $n, k \in \mathbb{N}$. All the sets $E=\bigcup\left\{E_{n}^{*}(k): n, k \in \mathbb{N}\right\} \in \mathcal{E}$ are of the additive Borel class $\alpha=\sup \{\alpha(n, k): n, k \in \mathbb{N}\}$. This concludes our proof.

Here we used implicitly the argument giving "discrete $\sigma$-decomposability" (or " $\sigma$-discrete decomposability" in other words) of point-countable families with a $\sigma$-discrete network which was observed by E. Michael ([53, Lemma 3.2]).

Theorem 2.31. Let $f: X \rightarrow Y$ be a Borel bimeasurable mapping of a completely metrizable space $X$ to a completely metrizable space $Y$.

(a) If $f$ takes open sets of some $\sigma$-discrete base $\mathcal{U}$ to an almost discretely $\sigma$ decomposable family, or

(b) if the fibers $f^{-1}(y), y \in Y$, are relatively compact in $X$,

then the images $f(G)$ of open sets $G \subset X$ are of a bounded Borel class.

The case (a) follows from the preceding lemma applied to $\mathcal{E}=f(\mathcal{U})$. We realize that $f$ has a Borel class by Theorem 2.10. Let $B^{*}$ be relatively closed in $f(B)$ for $B \in \mathcal{U}$. Then $\left\{B \cap f^{-1}\left(B^{*}\right) ; B \in \mathcal{U}\right\}$ is a $\sigma$-discrete family of Borel sets of a bounded class, so it is Borel additive. Thus $\left\{B^{*}=f\left(B \cap f^{-1}\left(B^{*}\right)\right): B \in \mathcal{U}\right\}$ is Borel additive and the assumptions of Lemma 2.30 are verified.

The case (b) can be reduced to (a). Consider any base $\mathcal{U}=\bigcup_{n \in \mathbb{N}} \mathcal{U}_{n}$ of $X$ such that each $\mathcal{U}_{n}$ is discrete. Then $f\left(\mathcal{U}_{n}\right)$ is Borel additive and point-finite. So by Lemma $2.7 f\left(\mathcal{U}_{n}\right)$ has a $\sigma$-discrete network, and so $f(\mathcal{U})$ is (almost) discretely $\sigma$-decomposable. We may apply (a) now.

Inspired by Theorem 2.22, we ask

Problem 2.32. Let $f: X \rightarrow Y$ be a Borel measurable mapping between completely metrizable spaces $X$ and $Y$. Is $f$ Borel bimeasurable if it takes $G_{\delta}$ sets in $X$ to Borel sets in $Y$ ?

It is natural to ask some supplementary questions:

Problem 2.33. Let $f: X \rightarrow Y$ be a Borel bimeasurable mapping between completely metrizable spaces $X$ and $Y$. Is it discrete-Borel bimeasurable? Is it Souslin bimeasurable?

Problem 2.34. Is there a mapping $\beta:\left[0, \omega_{1}\right) \rightarrow\left[0, \omega_{1}\right)$ such that the sets $E_{n}$ in the decomposition of Theorem 2.22 can be found in the Borel class $\beta(\alpha)$ if $f$ is Borel bimeasurable and of class $\alpha$ between completely metrizable spaces?

This seems to be open even for mappings between Polish spaces.

The statements [33, Proposition 4.1 and Theorem 4.4] give another result on preservation of Borel sets in a sense. It says, roughly speaking, that preservation of relatively Borel sets implies absolute Borelness of the range under some assumptions.

Theorem 2.35. Let $f: X \rightarrow Y$ be a (discrete-)Borel bimeasurable mapping of an absolute Borel set $X$ within metric spaces onto a metric-analytic space $Y$ which maps sets of any additive or multiplicative class $\gamma<\omega_{1}$ to sets of the corresponding class $\beta+\gamma$ for some $\beta<\omega_{1}$. Then $Y$ is absolute Borel in metric spaces. 
Indeed, the proof of [33, Proposition 4.1] works only with countable, and so Borel, classes in this case. The existence of a Borel class $\alpha<\omega_{1}$ of $f$ follows from Theorem 2.10. The statement of [33, Theorem 4.4] gives even some estimate on the class of $Y$. Since it is countable in our case, we conclude that $Y$ is Borel in its completion (cf. the definition of the "scattered-Borel classes" on pages 153-4 of the quoted paper and the remark about Borel classes following it).

It is not difficult to deduce from Theorem 2.27, (b) implies (c), or directly from [35, Theorem 3.1], that mappings with $\sigma$-compact fibers which fulfill (b) can be decomposed as in (c) with $E_{0}$ empty. There are similar theorems concerning decompositions of $\mathcal{F}_{\sigma}$ measurable mappings and of $\mathcal{F}_{\sigma}$ bimeasurable mappings due to Hansell, Jayne, and Rogers.

Theorem 2.36 ([46, Theorems 5]; proof of [45, Theorem 1], cf. [26, Theorem 3]).

(a) Let $f: X \rightarrow Y$ be a mapping between completely metrizable spaces $X$ and $Y$ such that $f^{-1}(F)$ is $\mathcal{F}_{\sigma}$ in $X$ for every $\mathcal{F}_{\sigma}$ set $F$ in $Y$, i.e. $f$ is $\mathcal{F}_{\sigma}$ measurable. Then there are closed sets $E_{n} \subset X$ such that the restrictions of $f$ to each $E_{n}$ are continuous.

(b) Let $f: X \rightarrow Y$ be an $\mathcal{F}_{\sigma}$ bimeasurable surjective mapping between metricanalytic spaces $X$ and $Y$ which takes discrete families to almost discretely $\sigma$-decomposable families. Then there are closed sets $E_{n} \subset X$ such that the restriction of $f$ to each $E_{n}$ is a closed continuous mapping.

Preservation of absolute Borel spaces was proved in [40, Theorem 4.2] for $\mathcal{F}_{\sigma}$ bimeasurable mappings without additional assumptions:

Theorem 2.37. Let $f: X \rightarrow Y$ be an $\mathcal{F}_{\sigma}$ bimeasurable mapping of an absolute Borel metrizable space $X$ onto a metric-analytic space $Y$. Then $Y$ is absolute Borel.

It seems to be of some interest whether some analogies for $\mathcal{M}_{\sigma}$ measurable or $\mathcal{M}_{\sigma}$ bimeasurable mappings hold as well if $\mathcal{M}$ is, e.g., a multiplicative Borel classe higher than $\mathcal{F}$.

The following interesting problem of Hansell, which is slightly related to the preceding once on the necessary conditions of some Souslin or discrete-Borel additive families, is still open.

Problem 2.38. Let $\mathcal{A}$ be a point-countable and Borel additive family, or discreteBorel additive family, of subsets of a completely metrizable space. Does $\mathcal{A}$ have a $\sigma$-discrete refinement?

Hansell showed in [20, Theorem 3.1(b)] that the conclusion is not provable for point-countable Souslin additive families in ZFC. Fleissner showed in [5] that under the consistency of $\left(\mathrm{SC}_{2}\right)$ the conclusion is true even for all point-countable Souslin additive families. There are some partial positive answers to this problem. The results of Hansell (in [21, Theorem 3.3] for point-countable $\mathcal{F}_{\sigma}$ additive families) and J. Spurný (in [60, Theorem 6] for $\mathcal{G}_{\delta}$ additive families) were recently improved by Spurný and Zelený [62, Theorem 1.1].

Theorem 2.39. Let $\mathcal{A}$ be a point-countable $\mathcal{F}_{\sigma \delta}$ additive family in a metric-analytic space. Then $\mathcal{A}$ has a $\sigma$-discrete refinement.

\section{EXtensions of THE DESCRIPTIVE SET THEORY TO TYCHONOFF SPACES}


3.1. Several types of completeness of Tychonoff spaces. Let us recall that several notions of completeness of Tychonoff spaces can be viewed as an absolute property (within Tychonoff spaces). We say that a Tychonoff space is absolute $\mathcal{P}$ if it is in $\mathcal{P}$ (or it has the property $\mathcal{P}$ ) in any Tychonoff space to which it is embedded.

Theorem 3.1. The following statements on a Tychonoff space $X$ are equivalent:

(a) $X$ is Čech-complete, i.e., $\mathcal{G}_{\delta}$ in its Stone-Čech compactification.

(b) $X$ is absolute $\mathcal{G}_{\delta}$ in its closure, i.e., it is absolutely an intersection of a closed and $a \mathcal{G}_{\delta}$ set.

(c) $X$ is $\mathcal{G}_{\delta}$ in its closure in a compact space.

(d) $X$ admits a complete sequence of open covers.

These results can be found in [4, Theorem 3.9.1]. The equivalence of (a) and (d) easily follows from [4, Theorem 3.9.2].

We call a Tychonoff space $X \mathcal{P}$-K-complete if $X$ admits a complete sequence of covers with the property $\mathcal{P}$. So (d) can be restated by saying " $X$ is open- $K$ complete".

The spaces of the next theorem form a larger class than Cech-complete spaces and are known under various names.

Let us recall that a family $\mathcal{D}$ of subsets of a Tychonoff space $X$ is scattered if it is disjoint and if every nonempty subfamily $\mathcal{E} \subset \mathcal{D}$ contains a nonempty element $E \in \mathcal{E}$ which is relatively open in $\bigcup \mathcal{E}$.

Theorem 3.2 ([38, Theorem 2.5]). The following statements on a Tychonoff space $X$ are equivalent:

(a) $X$ is absolute $\mathcal{H}_{\delta}$.

(b) $X$ is $\mathcal{H}_{\delta}$ in a compact space.

(c) $X$ admits a complete sequence of scattered covers, i.e., $X$ is scattered-Kcomplete.

A disjoint family $\mathcal{D}$ of subsets of a Tychonoff space is isolated (or relatively discrete) if each $D \in \mathcal{D}$ is relatively open in $\bigcup \mathcal{D}$. Thus we call a Tychonoff space isolated- $K$-complete if it admits a complete sequence of isolated covers. Naturally, isolated- $K$-complete spaces form a subclass of Čech-complete ones.

3.2. Absolute $\mathcal{S}(\mathcal{F}), \mathcal{S}(\mathcal{B})$, and $\mathcal{S}(\mathcal{H})$ spaces and complete sequences of covers. Absolute $\mathcal{S}(\mathcal{F})$ spaces are called $K$-analytic. This class was introduced and studied by several authors under several names using different descriptions which turned out to be equivalent [42] and [8, Theorem 9.3]. We shall mention the descriptions of G. Choquet, M. Sion, and Frolík in Section 3.4, which is devoted to parametrizations of generalized analytic spaces.

Theorem 3.3 ([8, Theorems 4.11 and 9.3]). The following properties of a Tychonoff space $A$ are equivalent:

(a) $A$ is an absolute $\mathcal{S}(\mathcal{F})$ space.

(b) $A$ is in $\mathcal{S}(\mathcal{F}(K))$ in a compact space $K$.

(c) $A$ is the projection of a Lindelöf Čech-complete subspace of $A \times \mathbb{N}^{\mathbb{N}}$.

(d) A admits a complete sequence of countable covers.

As a consequence of (c) we get the following two corollaries.

Corollary 3.4. Every K-analytic space is Lindelöf. 
Example 3.5. By M. Talagrand [64], if $X$ is a weakly compactly generated (WCG) Banach space and $\tau_{w}$ is its weak topology, then the space $\left(X, \tau_{w}\right)$ is $K$-analytic (even $K_{\sigma \delta}$ in $\left.\left(X^{* *}, w^{*}\right)\right)$. Using this, Talagrand showed that $\left(X, \tau_{w}\right)$, for a weakly compactly generated Banach space $X$, is Lindelöf. He also showed that there are non WCG spaces such that $\left(X, \tau_{w}\right)$ is Lindelöf. These results gave a full answer to a conjecture of H.H. Corson. Talagrand also posed the problem whether the $K$-analyticity of the weak topology of a Banach space implies that it is $K_{\sigma \delta}$ when embedded in the canonical way to the second dual with the weak star topology $w^{*}$. The negative answer to this question was given recently by S.A. Argyros, A.D. Arvanitakis, and S.K. Mercourakis in [1].

All the notions recalled so far bring a serious limitation to the case of Banach spaces endowed with their weak topology. The metric-analyticity, and so metrizability, implies that $X$ is finite-dimensional. Since $K$-analytic spaces are Lindelöf, only those weak topologies which are Lindelöf can be $K$-analytic. L. Vašák proved that weakly $K$-analytic Banach spaces (even weakly countably determined) have an equivalent Kadec norm. However, there are also nonseparable spaces with nonLindelöf weak topology with a Kadec norm. G.A. Edgar [3] showed that such spaces with their weak topology are Borel in the weak-star topology of their second dual (see Hansell [22] for further examples and references).

Example 3.6. If the Banach space $X$ admits an equivalent norm with the Kadec property, i.e., if the norm and the weak topologies coincide on the unit sphere, then $X$ endowed with its weak topology is Borel in $X^{* *}$ endowed with the weakstar topology $\tau_{w^{*}}$. In fact, it is of the form $\bigcap_{k \in \mathbb{N}} \bigcup_{n \in \mathbb{N}}\left(F_{k n} \cap G_{k n}\right)$, where $F_{k n} \in$ $\mathcal{F}\left(X^{* *}, w^{*}\right)$ and $G_{k n} \in \mathcal{G}\left(X^{* *}, w^{*}\right)([23$, Theorems 1.5 and 1.4(b)]). Thus it is absolute Borel. In fact $X$ is Borel in the $\sigma$-compact, and so absolute Borel, space $\left(X^{* *}, \tau_{w^{*}}\right)$. So it is absolute Borel by [58, Theorem 4.6, (i) implies (ii)], or also by [39, Corollary 14].

The class of absolute $\mathcal{S}(\mathcal{B})$ spaces was studied by Fremlin, see [43, 8. Appendix], under the name $\breve{C}$ ech-analytic spaces in an unpublished remark [6] and we have the following theorem. A family $\mathcal{I}$ is relatively open if each $I \in \mathcal{I}$ is relatively open in $\bigcup \mathcal{I}$. Countable unions of relatively open families are called $\sigma$-relatively open. Note that disjoint relatively open families are isolated (relatively discrete).

Theorem 3.7. The following properties of a topological space $A$ are equivalent:

(a) $A$ is an absolute $\mathcal{S}(\mathcal{B})$ space.

(b) $A$ is an $\mathcal{S}(\mathcal{B}(K))$ set in a compact space $K$.

(c) $A$ is the projection of a Čech-complete subspace of $A \times \mathbb{N}^{\mathbb{N}}$.

(d) A admits a complete sequence of $\sigma$-relatively open covers.

The equivalence of (a), (b), and (c) follows from [22, Theorem 5.3]. (The implication (b) implies (a) can be also proved using that continuous preimages preserve $\mathcal{S}(\mathcal{B})$ and that absolute $\mathcal{S}(\mathcal{B}(K))$ sets are preserved by perfect mappings, see [27] or [39, Corollary 13]). The equivalence of (c) and (d) was remarked by Frolík in [10], for its proof see, e.g., [32, Theorem 3].

Hansell introduced in [23], which appeared as a preprint already in 1989, the notion of "almost- $K$-analytic spaces" which we call "scattered- $K$-analytic" now, cf. [22], [29]. The class of these spaces is larger than that of Cech-analytic spaces and it turns out that it is more suitable for applications of some methods of the 
descriptive set theory. The equivalences below can be found in [31, Theorems 1 and $2]$ and [32, Theorem 1].

Theorem 3.8. The following properties of a topological space $A$ are equivalent:

(a) $A$ is an absolute $\mathcal{S}(\mathcal{H})$ space.

(b) $A$ is an $\mathcal{S}(\mathcal{H}(K))$ subset of a compact space $K$.

(c) $A$ is the projection of a scattered-K-complete subspace of $A \times \mathbb{N}^{\mathbb{N}}$.

(d) A admits a complete sequence of $\sigma$-scattered covers.

A topological space $(X, \tau)$ is $\sigma$-fragmented by a metric $\rho$ if, for every positive $\varepsilon$, there is a $\sigma$-scattered partition of $X$ to sets of $\rho$-diameter less than $\varepsilon$. We have the following relation between weakly scattered- $K$-analytic Banach spaces and $\sigma$ fragmentability.

Example 3.9 ([23, Theorem 1.10] and [32, Theorem 6]). Let $X$ be a Banach space. Then the following are equivalent:

(a) $\left(X, \tau_{w}\right)$ is scattered-K-analytic.

(b) $\left(X, \tau_{w}\right)$ is $\sigma$-fragmented by the norm of $X$.

(c) The identity of $(X,\|\cdot\|)$ onto $\left(X, \tau_{w}\right)$ maps discrete families to families with a $\sigma$-scattered network.

(d) $\left(X, \tau_{w}\right)$ has a $\sigma$-scattered network.

So $\sigma$-fragmentable Banach spaces with their weak topology are examples of scattered- $K$-analytic spaces.

Let us recall that a space is ( $\sigma$-isolated)- $K$-complete if it admits a complete sequence of $\sigma$-isolated covers. We have the following implication.

Theorem 3.10. If $A$ is the projection of an isolated- $K$-complete subspace of $A \times$ $\mathbb{N}^{\mathbb{N}}$, then $A$ is ( $\sigma$-isolated)-K-complete. The converse implication does not hold in general.

The implication can be found in [32, Theorem 2]. The counterexample was shown to me by Junnila and J. Pelant (the example can be found in [22, Example 6.22]).

We may ask the following question.

Problem 3.11. Does some descriptive property of sets characterize all ( $\sigma$-isolated)$K$-complete subspaces of compact spaces?

We see immediately that

\section{Theorem 3.12.}

(a) Every $K$-analytic space is ( $\sigma$-isolated)-K-complete.

(b) Every ( $\sigma$-isolated)-K-complete space is Čech-analytic.

(c) Every C̆ech-analytic space is scattered-K-analytic.

3.3. Descriptive properties of generalized bi-analytic sets. Frolík showed, using multivalued parametrizations of $K$-analytic spaces, that some methods of the classical descriptive set theory apply to $K$-analytic spaces. As an example we state a particular version of the separation principle. Let us recall that $B$ is a Baire set in $X$ if it belongs to the smallest $\sigma$-algebra containing zero sets of continuous functions.

Theorem 3.13. $A$ subset $B$ of a $K$-analytic space $A$ is a Baire set if and only if $B$ and $A \backslash B$ are $K$-analytic. 
It is still a challenge to answer satisfactorily the following question.

Problem 3.14. Is there some nontrivial characterization of spaces $X$ such that $X$ and $K \backslash X$ are Čech-analytic subspaces of some compact space $K$ ?

We may characterize the "bi- $\mathcal{S}(\mathcal{H})$ " subspaces, of a scattered- $K$-analytic space, as those which belong absolutely to the $\sigma$-algebra of scattered-Borel sets. By this we understand the smallest $\sigma$-algebra of subsets of $X$ which contains all Borel sets and which is closed with respect to the unions of scattered families of its elements.

Theorem 3.15 ([22, Theorem 6.28] and [29, Theorem 2]). B is scattered-Borel in a scattered-K-analytic space $A$ if and only if $B$ and $A \backslash B$ are scattered-K-analytic.

We have the following description of bi- $(\sigma$-isolated $)-K$-complete spaces. We define isolated-Borel sets as the elements of the smallest $\sigma$-algebra of subsets of $X$ which contains all Borel sets and which is closed with respect to the unions of isolated families of its elements.

Theorem 3.16. If $B \subset X$ and $X \backslash B$ are ( $\sigma$-isolated)-K-complete, then $B$ is isolated-Borel in $X$.

The proof can be received following closely that in [22, Theorem 6.28]. Let $\mathcal{C}_{n}$ and $\mathcal{D}_{n}$ be sequences of $\sigma$-isolated covers by $B$ and $X \backslash B$, respectively. We may assume without loss of generality that all $\mathcal{C}_{n}$ 's and $\mathcal{D}_{n}$ 's are partitions, since arbitrary refinements preserve completeness. We may also assume that $\mathcal{C}_{n+1}$ refines $\mathcal{C}_{n}$ and $\mathcal{D}_{n+1}$ refines $\mathcal{D}_{n}$ by considering $\left\{C_{1} \cap \cdots \cap C_{n}: C_{i} \in \mathcal{C}_{i}, i=1, \ldots, n\right\}$ instead of $\mathcal{C}_{n}$, and similarly for $\mathcal{D}_{n}$ 's. Assuming that $B$ and $X \backslash B$ cannot be separated by an isolated-Borel set, we deduce using [22, Lemma 6.27] that some pair of $C_{1} \in \mathcal{C}_{1}$ and $D_{1} \in \mathcal{D}_{1}$ cannot be separated. Using [22, Lemma 6.27] inductively to the pairs $C_{n}, D_{n}$, we get centered sequences $C_{n} \in \mathcal{C}_{n}$ and $D_{n} \in \mathcal{D}_{n}$ such that the pairs $C_{n}$ and $D_{n}$ cannot be separated for every $n \in \mathbb{N}$. However, the completeness implies that the closures of $C_{n}$ 's and of $D_{n}$ 's converge to disjoint compact sets, say $K$ and $L$ (see, e.g., [31, Proposition 4.1] for the last argument). These can be separated by disjoint open sets $G \supset K$ and $H \supset L$. However, for sufficiently large $n$ 's the closure of $C_{n}$ is a subset of $G$ and the closure of $D_{n}$ is a subset of $H$, so $C_{n}$ and $D_{n}$ are separated, a contradiction.

To get the converse implication, more exactly, to get that isolated-Borel sets (or even open sets only) are $(\sigma$-isolated)- $K$-complete in a $(\sigma$-isolated $)-K$-complete space, we need something more, e.g., that every family of open sets in $X$ has a $\sigma$-isolated refinement.

Example 3.17. $\left[0, \omega_{1}\right)$ is not ( $\sigma$-isolated)-K-complete, while it is, as an open set, isolated-Borel in $\left[0, \omega_{1}\right]$.

Let us derive it from the following auxiliary statement:

Lemma 3.18. If a stationary set $S \subset\left[0, \omega_{1}\right)$ is covered by a $\sigma$-isolated family $\mathcal{E}$ of subsets of $\left[0, \omega_{1}\right)$, then some $E \in \mathcal{E}$ is stationary.

Let us suppose the validity of the lemma and have a complete sequence of $\sigma$ isolated covers $\mathcal{C}_{n}$ of $\left[0, \omega_{1}\right)$. Put $C_{0}=\left[0, \omega_{1}\right)$ and suppose that we have $C_{0}, C_{1} \in$ $\mathcal{C}_{1}, \ldots, C_{n-1} \in \mathcal{C}_{n-1}$ for some $n \in \mathbb{N}$ such that $C_{0} \cap \cdots \cap C_{n}$ is stationary, in particular uncountable. Since every compact set in $\left[0, \omega_{1}\right)$ is countable and has a 
countable open neighbourhood, the sequence $\overline{C_{1} \cap \cdots \cap C_{n}}$ cannot converge to a compact subset of $\left[0, \omega_{1}\right)$, a contradiction (cf. [31, Proposition 4.1] again).

To prove Lemma 3.18 we realize first that we may assume without loss of generality that $\mathcal{E}$ is isolated and uncountable since countable unions of nonstationary sets are nonstationary due to the fact that countable intersections of closed unbounded sets are closed unbounded in $\left[0, \omega_{1}\right)$. We may thus assume that $\mathcal{E}=\left\{E_{\alpha}: \alpha<\omega_{1}\right\}$, where the relatively open sets $E_{\alpha}$ which form the cover of $S$ are nonempty and pairwise disjoint. We may find inductively $a_{\alpha}, s_{\alpha}, b_{\alpha} \in\left[0, \omega_{1}\right)$ for $\alpha<\omega_{1}$ such that

(a) $a_{\alpha}<s_{\alpha}<b_{\alpha}<a_{\beta}$ for $\alpha<\beta<\omega_{1}$, and

(b) $s_{\alpha} \in E_{\alpha}$.

Having it, we put $F=\overline{\left\{s_{\alpha}: \alpha<\omega_{1}\right\}} \backslash \bigcup\left\{\left(a_{\alpha}, b_{\alpha}\right): \alpha<\omega_{1}\right\}$. Note that $F$ is closed and since for every limit ordinal $\alpha<\omega_{1}$ the ordinal sup $\overline{\left\{s_{\beta}: \beta<\alpha\right\}}$ is greater than all $b_{\beta}, \beta<\alpha$, and at most equal to $a_{\alpha}, F$ is unbounded in $\left[0, \omega_{1}\right)$. Therefore there is an $s \in S \cap F$. It differs from all $s_{\alpha}$ and therefore it has in each its neighbourhood infinitely many $s_{\alpha}$ 's, which is a contradiction with the assumption that $\mathcal{E}$ is isolated in $S$.

Problem 3.19. Is it possible to characterize the $\sigma$-algebra of bi-( $\sigma$-isolated $)-K$ complete spaces as the smallest $\sigma$-algebra containing some type of sets and closed with respect to some operations?

3.4. Parametrizations of generalized analytic spaces. Now we state the equivalent parametrizations of $K$-analytic spaces mentioned in Section 3.2. $K$-analytic spaces were introduced by Choquet as continuous images of an $\mathcal{F}_{\sigma \delta}$ subspace of a compact space, by Sion as continuous images of a $K_{\sigma \delta}$ subspaces of a space, by Frolík as images of Polish spaces under upper semicontinuous compact-valued mappings, and also as spaces admitting a complete sequence of countable covers. A mapping $f$ which takes values of $X$ to compact sets in $Y$ is called upper semicontinuous and compact-valued if the sets $\{x \in M: f(x) \subset G\}$ are open for open sets $G$ in $Y$. We abbreviate this property of set-valued mappings to usc-K. Let us state some characterizations explicitly.

Theorem 3.20. The following properties of a topological space A are equivalent:

(a) $A$ is an absolute $\mathcal{S}(\mathcal{F})$ space.

(b) $A$ is a continuous image of a Lindelöf $\check{C}$ ech-complete space.

(c) $A$ is the image of a Polish space under a usc-K mapping.

The equivalence of (a) and (c) can be found in [8, Theorem 4.11]. This together with the equivalence of (b) can be found in [44, Theorem 2.8.1] or also in [22, Theorem 3.1].

Realizing that the composition of a continuous and an usc- $K$ mapping (or of two usc- $K$ mappings) is usc- $K$ again, we get from (c) above

Corollary 3.21. Continuous (or usc-K) images of $K$-analytic spaces are $K$-analytic.

Čech-analytic spaces are projections of Čech-complete spaces along $\mathbb{N}^{\mathbb{N}}$ by Theorem 3.7.

Problem 3.22. Is there some property of families of sets such that continuous mappings preserving that property of families preserve Čech-analytic spaces?

As we already noted, scattered- $K$-analytic spaces are better suited for applying the methods of the descriptive set theory. 
Theorem 3.23. The following properties of a topological space $A$ are equivalent:

(a) $A$ is absolute $\mathcal{S}(\mathcal{H})$.

(b) $A$ is the image of a completely metrizable space $M$ (which is a closed subset of $D^{\mathbb{N}}$ for some discrete $D$ ) under a usc-K mapping which preserves families having a $\sigma$-scattered refinement.

(c) A is the image of a completely metrizable space $M$ (which is a closed subset of $D^{\mathbb{N}}$ for some discrete $D$ ) under a usc-K mapping which preserves pointcountable indexed families having a $\sigma$-scattered network.

A proof of the equivalence of (a) and (c) follows from [32, Theorem 1] and Theorem 2.3. The condition (b) is weaker than (c) and the implication (b) implies (a) follows from [31, Theorem 1, (c) implies (d)].

By (b) we immediately get

Corollary 3.24. Images of scattered-K-analytic spaces by continuous (or usc- $K$ ) mappings which preserve families with a $\sigma$-scattered refinement are scattered-Kanalytic.

We may define isolated-K-analytic spaces (the terminology follows [22] and [29]) as the images of completely metrizable spaces by usc- $K$ mappings which preserve point-countable indexed families with a $\sigma$-isolated network. This class of spaces was independently introduced by Frolík [10] and by Hansell [23] (who called them descriptive- $K$-spaces). It is obviously preserved by continuous (or only usc- $K$ ) mappings which preserve point-countable indexed families with a $\sigma$-isolated network.

Example 3.25 ([23, Theorem 1.5]). All Banach spaces admitting an equivalent Kadec norm are isolated-K-analytic.

Theorem 3.26. Let us consider the following statements:

(a) $A$ is isolated-K-analytic.

(b) $A$ is ( $\sigma$-isolated)-K-complete (it admits a complete sequence of $\sigma$-isolated covers.

(c) $A$ is the projection of an isolated-K-complete subspace of $A \times \mathbb{N}^{\mathbb{N}}$.

Then (a) implies (b), (c) implies (a), and the implication (b) implies (c) does not hold in general.

The positive results can be found in [32, Theorem 2]. The already mentioned example [22, Example 6.22] gives the negative statement. It seems that the following question may still have a positive answer.

Problem 3.27. Does (a) imply (c) in the above theorem?

3.5. Preservation of generalized analytic spaces. We saw already as immediate Corollaries 3.21 and 3.24 of some parametrizations of the corresponding spaces, or of the very definition of isolated- $K$-analytic spaces above that these types of spaces are preserved by continuous (or usc- $K$ ) mappings with the relevant property of preservation of some kind of families. We could again ask what additional property, in place of the mentioned preservation of suitable families, in the last two cases is necessary. Since many questions are open even for metrizable spaces (at least in ZFC), we limit ourselves to one-to-one mappings, which are fully understood in the metrizable case. 
Problem 3.28. Let $f: X \rightarrow Y$ be a continuous one-to-one mapping between compact spaces $X$ and $Y$ which takes $\mathcal{S}(\mathcal{H})$ sets (or isolated-K-analytic subspaces) to $\mathcal{S}(\mathcal{H})$ sets (or to isolated-K-analytic subspaces). Does $f$ preserve families with a $\sigma$-scattered refinement (or $\sigma$-isolated refinement)?

We saw in the metrizable case that the investigation of necessary conditions for bimeasurability lead to the study of suitably additive families of sets. We may again note that $f$ as in the problem takes scattered (isolated) families of $\mathcal{S}(\mathcal{H}$ ) sets (isolated- $K$-analytic sets) to $\mathcal{S}(\mathcal{H})$ (isolated- $K$-analytic) additive disjoint families. Thus the problem in other words consists in the question whether such additive disjoint families have a $\sigma$-scattered (or $\sigma$-isolated) refinement. The only positive results in this direction that we know so far are proved in [30, Theorem 2] under the assumption that there is no (two-valued) measurable cardinal.

Theorem 3.29 (nonexistence of a measurable cardinal).

(a) Let $\mathcal{A}$ be a disjoint scattered-K-analytic additive family in a Tychonoff space $X$. Then $\mathcal{A}$ has a $\sigma$-scattered network.

(b) Let $\mathcal{A}$ be a disjoint isolated-K-analytic additive family in a Tychonoff space $X$. Then $\mathcal{A}$ has a $\sigma$-isolated network.

We however do not know the answers to the following questions. The first one is just a reformulation of the preceding problem.

\section{Problem 3.30.}

(a) Can it be proved in $Z F C$ that every $\mathcal{S}(\mathcal{H})$ (or isolated-K-analytic) additive family of pairwise disjoint sets in a compact space have a $\sigma$-scattered (or $\sigma$-isolated) network?

(b) Can the same be proved if the family is not pairwise disjoint but just pointfinite even under the nonexistence of the measurable cardinal?

Related questions concerning just $\mathcal{S}(\mathcal{F})$ additivity are much easier to handle:

Theorem 3.31 ([12, Theorem 1]). Let $f: M \rightarrow X$ be an usc-K mapping of a complete metric space $M$ to a Tychonoff space $X$. If $\mathcal{A}$ is a point-finite $\mathcal{S}(\mathcal{F}(X))$ additive family in $X$, then the family $\left\{f^{-1}(A)=\{t \in M: f(t) \cap A \neq \emptyset\}: A \in \mathcal{A}\right\}$ indexed by $A$ 's is point-countable and has a $\sigma$-discrete network.

As a corollary we get using the corresponding parametrizations:

\section{Corollary 3.32.}

(a) Point-finite $\mathcal{S}(\mathcal{F})$ additive families in $K$-analytic spaces are countable.

(b) Point-finite $\mathcal{S}(\mathcal{F})$ additive families in isolated-K-analytic spaces have a $\sigma$ isolated network.

(c) Point-finite $\mathcal{S}(\mathcal{F})$ additive families in scattered-K-analytic spaces have a $\sigma$-scattered network.

The Hansell's theorem on point-countable $\mathcal{F}_{\sigma}$ additive families has the following topological variant by Spurný ([61, Theorem 4.2]):

Theorem 3.33. Let $\mathcal{E}$ be a point-countable $\mathcal{F}_{\sigma}$ additive cover of a scattered- $K$ analytic space. Then $\mathcal{E}$ has a $\sigma$-scattered refinement.

We do not have any analogy of Theorem 2.15 which implies that the metricanalytic spaces are preserved by perfect mappings. Therefore the next results (see 
[39, Corollaries 7 and 13] for both of them, the second one was proved already in [27]) may be useful.

Theorem 3.34. Let $f: X \rightarrow Y$ be a perfect mapping (continuous closed with compact fibers) of $X$ onto $Y$.

(a) If $X$ is scattered- $K$-analytic then $Y$ is also scattered-K-analytic.

(b) If $X$ is Čech-analytic then $Y$ is also Čech-analytic.

So far we discussed the preservation of our generalized analytic spaces by some continuous (or usc- $K$ ) mappings. The natural question about suitably measurable mappings $f: X \rightarrow Y$ seems to be not so easy to answer. The standard method of studying the restriction of the continuous projection to $Y$ of the graph $G$ of $f$ needs to derive the appropriate analyticity of $G$. Thus using this method we can study only mappings to metrizable spaces (or to spaces with suitable networks).

For $K$-analytic spaces we have a result of Frolík [9, Theorem 1$]$ :

Theorem 3.35. Let $X$ be $K$-analytic, $Y$ be metrizable, and $f: X \rightarrow Y$ be $\mathcal{S}(\mathcal{F})$ measurable (or just Baire measurable). Then the graph of $f$ and $f(X)$ are $K$ analytic. If $f^{-1}(B)$ is Baire in $X$, then $B$ is Baire in $f(X)$.

Note that the preimages of Baire, or even all bi-Souslin, subsets of $Y$ are biSouslin, and therefore Baire by the separation principle (Theorem 3.13).

In [37, Theorem 2.8] we use scattered-analytic for scattered- $K$-analytic with a $\sigma$-scattered network and isolated-analytic for isolated- $K$-analytic (equivalently, $(\sigma$ isolated)- $K$-complete in this case) with a $\sigma$-isolated network. These classes of spaces were introduced in [23] under the name almost descriptive (descriptive) spaces and our terminology can be found in later papers, e.g., [22] or [29]. See also Theorem 3.45 and the references below.

Theorem 3.36 ([37, Theorem 2.8]). Let $X$ and $Y$ be scattered-analytic, $f: X \rightarrow$ $Y$ be scattered-analytic measurable, and $f$ preserves the point-countable indexed families with a $\sigma$-scattered network. Then the graph of $f$ and $f(X)$ are scatteredanalytic.

If we replace "scattered" by "isolated", the statement remains valid.

The analogical result with $X$ just scattered- $K$-analytic and the mapping $\mathcal{S}(\mathcal{H})$ measurable can be proved following the proofs in the quoted paper. It would be nice to prove analogical result for more general spaces $Y$.

The "separation" theorems give possibilities to get information about the descriptive properties of the images of some continuous mappings to Tychonoff spaces and some measurable mappings to metrizable spaces again. The case of $K$-analytic (and "K-Luzin spaces") is fully understood (see [8, Theorem 7.7]). We are not going to discuss the other cases here. There are however some results concerning Borel sets which are of different flavour and we point out some of them.

3.6. Preservation of Borel sets. M. Raja studied in [58] the description of absolute Borel spaces. The key notion of his study is the notion of a nicely behaving subclass of Borel measurable mappings called p-Borel mappings. Let us recall that $f: X \rightarrow Y$ is $p$-Borel if $f^{-1}(\mathcal{G}(Y))$ has a network $\mathcal{N}$ of the form $\mathcal{N}=\left\{B_{n} \cap U: U \in \mathcal{G}(X), n \in \mathbb{N}\right\}$ for some Borel sets $B_{n}$ in $X$. He shows that absolute Borel spaces are characterized by continuous parametrizations ([58, Theorem 4.6]): 
Theorem 3.37. A Tychonoff space $B$ is absolute Borel if and only if there is a Cech-complete space $C$ and a continuous bijective mapping $f: C \rightarrow B$ with a $p$-Borel inversion $f^{-1}$.

As a consequence of his investigation Raja obtains the following result on preservation in [58, Theorem 4.13]:

Theorem 3.38. If $X$ is absolute Borel and $f: X \rightarrow Y$ is a bijection of $X$ to $Y$ such that both $f$ and $f^{-1}$ are $p$-Borel, then $Y$ is absolute Borel.

We already referred in Example 3.6 above to another result of [58, Theorem 4.6]:

Theorem 3.39. A Tychonoff space is absolute Borel if and only if it is Borel in a compact space. Consequently, all Borel subsets of an absolute Borel space are absolute Borel.

The results of Holický and Spurný in [39] are of a different nature. They are concerned with preservation by perfect mappings. We already referred to another possibility of getting the preceding theorem using them. As an immediate consequence of [39, Corollary 15] we get the following result:

Theorem 3.40. Absolute Borel spaces are preserved by perfect mappings.

We have an elementary observation related to the Borel bimeasurability of particular mappings. It gives a well known example which concerns the norm and the week topologies of Banach spaces. We use that isolated (relatively discrete) families of Borel sets of a bounded class have Borel unions (see [23, Lemma 3.3], or also [22, Theorem 6.2], for the exact statement with the modified definition of Borel classes for nonmetrizable topological spaces). We formulate the result and an example below.

Lemma 3.41. Let $\mathcal{E} \subset \mathcal{A}$, where $\mathcal{A}$ is an additive Borel class containing open and closed sets in $X$. If $\mathcal{E}$ has a $\sigma$-isolated refinement $\mathcal{N}$, then $\bigcup \mathcal{E}$ is in $\mathcal{A}$.

Let $\mathcal{N}=\bigcup_{n \in \mathbb{N}} \mathcal{N}_{n}$, where each $\mathcal{N}_{n}$ is isolated. Put $\widehat{N}=\bar{N} \backslash \overline{\bigcup\left\{N^{\prime} \in \mathcal{N}_{n}: N^{\prime} \neq N\right\}}$. We choose an $E(N) \in \mathcal{E}$ such that $N \subset E(N)$ for every $N \in \mathcal{N}$. Then the family $\widehat{N} \cap E(N)$ is $\sigma$-isolated and consists of Borel sets of class $\mathcal{A}$. Therefore $\bigcup \mathcal{E}=\bigcup\{E(N): N \in \mathcal{N}\} \in \mathcal{A}$.

Proposition 3.42. Let $f: X \rightarrow Y$ be a mapping between topological spaces $X$ and $Y$ which takes elements of some open base $\mathcal{U}$ of $X$ to sets of a fixed family $\mathcal{A}$, which is closed with respect to the unions of $\sigma$-isolated subfamilies, to finite intersections, and contains closed and open sets (e.g., an additive Borel class). Let $f(\mathcal{U})$ have a $\sigma$-isolated network $\mathcal{N}$. Then $f(G)$ is in $\mathcal{A}$ for every open set $G \subset X$. In particular, if $f$ is one-to-one and $\mathcal{A}$ is a Borel class, then $f$ is Borel bimeasurable.

Let $G \subset X$ be open. Then $f(G)=\bigcup f\left(\mathcal{U}_{G}\right)$, where $\mathcal{U}_{G}=\{U \in \mathcal{U}: U \subset G\}$. The family $f\left(\mathcal{U}_{G}\right)$ belongs to $\mathcal{A}$ and has a $\sigma$-isolated refinement. By Lemma 3.41 above, its union $f(G)$ is in $\mathcal{A}$.

The particular case of the identity mapping of a Banach space $(X,\|\cdot\|)$ onto $\left(X, \tau_{w}\right)$ is of particular interest. There are further results on the coincidence of Borel sets in the norm and weak topologies, see [55, Proposition 1.5] or [56, Corollary 2.7].

Problem 3.43. Let $X$ be a Banach space such that $\left(X, \tau_{w}\right)$ is scattered-K-analytic. Is it true that every Borel set in the norm of $X$ is weakly Borel? 
A question of J. Oncina whether a Banach space in which the $\sigma$-algebras of norm and weak Borel sets coincide are necessarily scattered-analytic was answered by W. Marciszewski and Pol [52]. They showed that the negative answer is consistent with ZFC.

Problem 3.44. Is there an example in ZFC of a Banach space which is not scattered-analytic in the weak topology such that the Borel $\sigma$-algebras in the norm and weak topologies coincide?

3.7. Subclasses of absolute $\mathcal{S}(\mathcal{H})$ spaces. It would be optimal to have a theory for a class containing the others (this could be the class of absolute $\mathcal{S}(\mathcal{H})$, i.e., scattered- $K$-analytic, spaces among those we studied here), to distinguish the smaller classes by some characteristic properties and derive results on them using these properties and facts on the largest class. However, this seems to be hopeless. There are only a few results distinguishing smaller classes in the larger once in this spirit, and it concerns rather some better behaving proper subclasses of the above mentioned absolute $\mathcal{S}(\mathcal{H})$ or isolated- $K$-analytic spaces.

\section{Theorem 3.45.}

(a) If $A$ is scattered-K-analytic with a $\sigma$-scattered network ( $A$ is scatteredanalytic), then $X$ is the image of a complete metric space under a continuous mapping which preserves point-countable indexed families with a $\sigma$-scattered network. Equivalently, $X$ admits a complete sequence of $\sigma$ scattered networks.

(b) If $A$ is scattered-K-analytic with a $\sigma$-isolated network, then $X$ is the image of a complete metric space under a continuous mapping which preserves point-countable indexed families with a $\sigma$-isolated network ( $A$ is isolatedanalytic). Equivalently, $X$ admits a complete sequence of $\sigma$-isolated networks.

(c) If $A$ is scattered-K-analytic and has a countable network, then $X$ is a continuous image of a Polish space (such topological spaces are often called "analytic" even if nonmetrizable). Equivalently, $X$ admits a complete sequence of countable networks.

(d) If $A$ is scattered-K-analytic and separable metrizable, then it is analytic.

The proofs maybe deduced from [32, Theorem 5 and Proposition 5].

Example 3.46. If a Banach space $X$ admits an equivalent norm with the Kadec property, then the weak topology on $X$ has a $\sigma$-isolated network ([23, Theorem 1.5]).

By [23, Theorem 1.2] such spaces admit weakly $\sigma$-isolated network for the norm open sets. It follows that they admit a complete sequence of $\sigma$-isolated networks. In particular, the weak topology of any weakly $K$-analytic Banach space (or of any Eberlein compact space) is isolated-analytic. These and other examples are discussed in [23] after Theorem 1.5.

The problem on distinguishing $K$-analytic spaces among Cech-analytic spaces goes back to Fremlin who received a partial answer to it (see [43, 8. Appendix]):

Theorem 3.47. Let $X$ be Čech-analytic and hereditarily Lindelöf. Then $X$ is $K$-analytic.

We might prove this theorem using Theorem 3.7. There is a complete sequence of $\sigma$-relatively open covers $\mathcal{C}_{n}$ of $X$. Since $X$ is hereditarily Lindelöf, each $\mathcal{C}_{n}$ has 
a countable refinement (subcover). Thus we get a complete sequence of countable covers and use Theorem 3.3.

Problem 3.48. Is there some nontrivial characterization of Čech-analytic spaces among all scattered-K-analytic spaces?

The same for $K$-analytic spaces among all Čech-analytic, or all scattered-Kanalytic, spaces?

Some results, e.g., for mappings between isolated-Luzin or scattered-Luzin spaces, can be deduced from those on mappings between complete metric spaces (cf. [37, Theorem 3.1] about uniformization, [37, Theorem's 5.2 and 5.4 ] on "generalized projections", or [37, Theorem's 6.1 and 6.2 ] on fibers of bimeasurable mappings).

The author thanks J. Spurný and O. Kalenda for their help during the preparation of this text.

\section{REFERENCES}

[1] S.A. Argyros, A.D. Arvanitakis, and S.K. Mercourakis, Talagrand's $K_{\sigma \delta}$ problem, Topology Appl. 155 (2008), pp. 1737-1755.

[2] C. Dellacherie Un cours sur les ensembles analytiques, Analytic sets, Academic Press, London 1980, pp. 183-316.

[3] G.A. Edgar, Measurability in a Banach space, Indiana Univ. Math. J. 26 (1977), pp. 663-677.

[4] R. Engelking, General Topology, Heldermann Verlag, Berlin 1989.

[5] W.G. Fleissner, An axiom for nonseparable Borel theory, Trans. Amer. Math. Soc. 251 (1979), pp. 309-328.

[6] D.H. Fremlin, Čech-analytic spaces, unpublished note, 1980.

[7] D.H. Fremlin, R.W. Hansell, H.J.K. Junnila, Borel functions of bounded class, Trans. Amer. Math. Soc. 277 (1983), 835-849.

[8] Z. Frolík, A survey of separable descriptive theory of sets and spaces, Czechoslovak Math. J. 20 (1970), pp. 406-467.

[9] Z. Frolík, A measurable map with analytic domain and metrizable range is quotient, Bull. Amer. Math. Soc. 76 (1970), 1112-1117.

[10] Z. Frolík, Distinguished subclasses of Čech-analytic spaces, Comment. Math. Univ. Carol. 25 (1984), pp. 368-370.

[11] Z. Frolík, On restrictions of projections along separable metric spaces, Report of the Department of Mathematics and Informatics 85-42, Delft University of Technology, Delft 1985

[12] Z. Frolík and P. Holický Decomposability of completely Suslin-additive families, Proc. Amer. Math. Soc. 82 (1981), pp. 359-365.

[13] Z. Frolík and P. Holický Applications of Luzinian separation principles (non-separable case), Fund. Math. 117 (1983), pp. 165-185.

[14] Z. Frolík and P. Holický, Analytic and Luzin spaces (non-separable case), Topology Appl. 19 (1985), pp. 129-156.

[15] R.W. Hansell, Borel measurable mappings for nonseparable metric spaces, Trans. Amer. Math. Soc. 161 (1971), pp. 145-169.

[16] R.W. Hansell, On the non-separable theory of $k$-Borel and $k$-Souslin sets, General Topology and Appl. 3 (1973), pp. 161-195.

[17] R.W. Hansell, On characterizing non-separable analytic and extended Borel sets as types of continuous images, Proc. London Math. Soc. 28 (1974), pp. 683-699.

[18] R.W. Hansell, On Borel mappings and Baire functions, Trans. Amer. Math. Soc. 194 (1974), pp. 195-211.

[19] R.W. Hansell, Point-finite Borel-additive families are of bounded class, Proc. Amer. Math. Soc. 83 (1981), 375-378.

[20] R.W. Hansell, Point-countable Souslin-additive families and $\sigma$-discrete reductions, General Topology and its Relations to Modern Analysis and Algebra. V. (Proceedings of 
the Fifth Prague Topological Symposium, 1981), Sigma Series in Pure Mathematics, 3. Heldermann Verlag, Berlin 1983, 254-260.

[21] R.W. Hansell, $F_{\sigma}$-set covers of analytic spaces and first class selectors, Proc. Amer. Math. Soc. 96 (1986), 365-371.

[22] R.W. Hansell, Descriptive topology, Recent Progress in General Topology, NorthHolland, Amsterdam 1992, pp. 275-315.

[23] R.W. Hansell, Descriptive sets and the topology of nonseparable Banach spaces, Serdica Math. J. 27 (2001), pp. 1-66.

[24] R.W. Hansell, J.E. Jayne, and C.A. Rogers, K-analytic sets, Mathematika 30 (1983), pp. $189-221$.

[25] R.W. Hansell, J.E. Jayne, and C.A. Rogers, Separation of K-analytic sets, Mathematika 32 (1985), pp. $147-190$.

[26] R.W. Hansell, J.E. Jayne, and C.A. Rogers, Piece-wise closed functions and almost discretely $\sigma$-decomposable families, Mathematika 32 (1985), pp. 229-247.

[27] R.W. Hansell and S. Pan, Perfect images of Čech-analytic spaces, Proc. Amer. Math. Soc. 123 (1995), pp. 293-298.

[28] P. Holický, Zdeněk Frolik and the descriptive theory of sets and spaces, Acta Univ. Carol. Math. Phys. 32 (1991), pp. 5 - 21.

[29] P. Holický, Čech analytic and almost K-descriptive spaces, Czech. Math. J. 43 (1993), pp. $451-466$.

[30] P. Holický, Borel maps with the "point of continuity property" and completely Borel additive families in some nonmetrizable spaces, Proc. Amer. Math. Soc. 120 (1994), pp. $951-958$.

[31] P. Holický, Luzin theorems for scattered-K-analytic spaces and Borel measures and Borel measures on them, Atti Sem. Mat. Fis. Univ. Modena XLIV (1996), pp. 395 - 413.

[32] P. Holický, Generalized analytic spaces, completeness and fragmentability, Czech. Math. Journal 51 (2001) pp. $791-818$.

[33] P. Holický, Extensions of Borel measurable maps and ranges of Borel bimeasurable maps Bulletin of the Polish Academy of Sciences. Mathematics 52 (2004), pp. $151-167$.

[34] P. Holický, Borel sets with countable sections for nonseparable spaces Proc. Amer. Math. Soc. 134 (2006), pp. 1519-1525.

[35] P. Holický, Borel sets with $\sigma$-compact sections for nonseparable spaces, Fund. Math. 199 (2008), 139-154.

[36] P. Holický, Decompositions of Borel bimeasurable mappings between complete metric spaces, Topology Appl. 156 (2008), pp. 217-226.

[37] P. Holický and V. Komínek, Descriptive properties of mappings of nonseparable Luzin spaces, Czechoslovak Math. J. 57 (2007), pp. 201-224.

[38] P. Holický and J. Pelant, Internal descriptions of absolute Borel classes, Topology Appl. 141 (2004), pp. $87-104$.

[39] P. Holický and J. Spurný, Perfect images of absolute Souslin and absolute Borel Tychonoff spaces, Topology Appl. 131 (2003), pp. 281 - 294.

[40] P. Holický and J. Spurný, $F_{\sigma}$-mappings and the invariance of absolute Borel classes, Fund. Math. 182 (2004), pp. $193-204$.

[41] P. Holický and M. Zelený, A converse of the Arsenin-Kunugui theorem on Borel sets with $\sigma$-compact sections, Fund. Math. 165 (2000), pp. $191-202$.

[42] J.E. Jayne, Structure of analytic Hausdorff spaces, Mathematika 23 (1976), no. 2, 208211.

[43] J.E. Jayne, I. Namioka, and C.A. Rogers, Topological properties of Banach spaces, Proc. London Math. Soc 66 (1993), pp. 651-672.

[44] J.E. Jayne and C.A. Rogers, K-analytic sets, Analytic Sets, Academic Press, London 1980, pp. $1-181$.

[45] J.E. Jayne and C.A. Rogers, Piece-wise closed functions, Math. Ann. 255 (1981), 499518.

[46] J.E. Jayne and C.A. Rogers, First level Borel functions and isomorphisms, J. Math. Pures Appl. 61 (1982), 177-205.

[47] J. Kaniewski and R. Pol, Borel-measurable selectors for compact-valued mappings in the non-separable case, Bull. Acad. Polon. Sci. 23 (1975), pp. 1043-1050.

[48] A.S. Kechris, Classical Descriptive Set Theory, Springer-Verlag, New York 1995. 
[49] K. Kuratowski, Topology, Vol. I, Academic Press, New York 1966.

[50] A. Louveau, A separation theorem for $\Sigma_{1}^{1}$ sets, Trans. Amer. Math. Soc. 260 (1980), 363-378.

[51] A. Louveau, Ensembles analytiques et boréliens dans les espaces produits, Astérisque, Soc. Math. de France 78 (1980).

[52] W. Marciszewski and R. Pol, On Banach spaces whose norm-open sets are $F_{\sigma}$-sets in the weak topology, J. Math. Anal. Appl. 350 (2009), pp. 708-722.

[53] E. Michael, On maps related to $\sigma$-locally finite and $\sigma$-discrete collections of sets, Pacific J. Math. 98 (1982), 139-152.

[54] Y.N. Moschovakis, Descriptive Set Theory, North-Holland, Amsterdam 1980.

[55] L. Oncina, The JNR property and the Borel structure of a Banach space, Serdica Math. J. 26 (2000), 13-32.

[56] L. Oncina and M. Raja, Descriptive compact spaces and renorming, Studia Math. 165 (2004), 39-52.

[57] D. Preiss, Completely additive disjoint system of Baire sets is of bounded class, Comment. Math. Univ. Carolinae 15 (1974), 341-344.

[58] On some class of Borel measurable maps and absolute Borel topological spaces, Topol. Appl. 123 (2002), 267-282.

[59] J. Saint-Raymond, Boréliens á coupes $\mathcal{K}_{\sigma}$, Bull. Soc. Math. France 104 (1976), 389-406.

[60] J. Spurný, G-delta-additive families in absolute Souslin spaces and Borel measurable selectors, Topology Appl. 154 (2007), 2779-2785.

[61] J. Spurný, F-sigma-additive covers of Čech complete and scattered-K-analytic spaces, Fund. Math. 199 (2008), 131-138.

[62] J. Spurný and M. Zelený, Additive families of low Borel classes and Borel measurable selectors, to appear in Canad. Math. Bull.

[63] S.M. Srivastava, A Course on Borel Sets, Springer-Verlag, New York, 1998.

[64] M. Talagrand, Sur une conjecture de H. H. Corson, Bull. Sci. Math. 99 (1975), pp. $211-212$.

Charles University, Faculty of Mathematics and Physics, Department of Mathematical Analysis, 18675 Prague 8, Czech Republic

E-mail address: holicky@karlin.mff.cuni.cz 